\title{
NUMERICAL ANALYSIS OF THE FLOWFIELD IN A SUPERSONIC COIL WITH AN INTERLEAVED JET CONFIGURATION AND ITS EFFECT ON THE GAIN DISTRIBUTION
}

\author{
Zong-min $\mathrm{Hu}^{* \#}$, Rho-shin Myong*+, Anh-thi Nguyen*, Zong-lin Jiang* and Tae-hwan Cho* \\ * Research Center for Aircraft Parts Technology and School of Mechanical and Aerospace Engineering, \\ Gyeongsang National University, Jinju 660-701, South Korea \\ \# LHD Laboratory, Institute of Mechanics, Chinese Academy of Sciences, Beijing 100080, China \\ ${ }^{+}$E-Mail: myong@gnu.ac.kr (Corresponding Author)
}

\begin{abstract}
In a supersonic chemical oxygen-iodine laser (COIL) operating without primary buffer gas, the features of flowfield have significant effects on the Laser efficiency and beam quality. In this paper three-dimensional, multispecies, chemically reactive CFD technology was used to study the flowfield in mixing nozzle implemented with a supersonic interleaving jet configuration. The features of the flowfield as well as its effect on the spatial distribution of small signal gain were analyzed.
\end{abstract}

Keywords: COIL without buffer, interleaved jet, flowfield, small signal gain

\section{INTRODUCTION}

Chemical oxygen-iodine laser (COIL) is a highpower chemical laser device that operates on a near infrared radiation $(\lambda=1.315 \mu \mathrm{m})$ of atomic iodine. The laser medium, $I^{*}$, is produced through a pumping reaction between singlet oxygen, $\mathrm{O}_{2}\left({ }^{1} \Delta\right)$, and ground state iodine, I. Research on COILs has accelerated owing to the potential military and industrial applications. Recently, a new concept of COIL operating without primary buffer gas was proved to be of higher efficiency and stability, and more applicability for mobile integration. This kind of COIL uses nitrogen rather than helium as the buffer gas in the secondary flow (Furman, Barmashenko and Rosenwaks, 1998; Furman et al., 2001; Fang, Sang and Chen, 2002 \& 2003), which results in characteristic velocity reduction. A series of experimental studies were completed with a supersonic COIL at Ben-Gurion University (Furman, Barmashenko and Rosenwaks, 1998; Furman et al., 2001; Bruins et al., 2002; Rybalkin et al., 2002). They concluded that the gain distribution is often inhomogeneous because of inefficient mixing (Rybalkin et al., 2002). Transverse jets in the transonic or supersonic section chock the nozzle flow and induce complicated flow discontinuities, which may in turn significantly affect the mixing and pumping processes.
In this paper, a new jet configuration called transverse interleaving jet was proposed and applied to a primary-buffer-free COIL. Then, threedimensional (3D) CFD technology was used to evaluate the flowfield and its effect on the gain field in the mixing nozzle.

\section{NUMERICAL MODELS AND ALGORITHMS}

\subsection{Governing equations and numerical models}

The COIL flow with a low density and Reynolds number from $10^{2}$ (based on the jet flow conditions and the orifice) to $10^{3}$ (based on the primary flow conditions and the inlet chamber size) is supposed to be governed by 3D Navier-Stokes equations. In Cartesian coordinates, the N-S equation for multicomponent, chemically reactive system is written as:

$$
\frac{\partial U}{\partial t}+\frac{\partial F_{i}}{\partial x_{i}}=\frac{\partial D_{i}}{\partial x_{i}}+S \quad(i=1,2,3)
$$

Here, $U$ is the unknown variable vector, $F_{i}(i=1,2,3)$ are convective flux vectors in $x_{i}(\mathrm{x}, \mathrm{y}$ and $\mathrm{z}$ ) directions, $D_{i}$ are the dissipation flux vectors, and $S$ is the chemical reaction source term, where 


$$
\begin{aligned}
& U=\left[\begin{array}{llllllll}
\rho_{1} & \rho_{2} & \cdots & \rho_{n s} & \rho u_{1} & \rho u_{2} & \rho u_{3} & \rho e
\end{array}\right] \\
& F_{i}=\left[\begin{array}{lllll}
\rho_{1} u_{i} & \rho_{2} u_{i} & \cdots & \rho_{n s} u_{i} & \rho u_{i} u_{1}+\delta_{i, 1} p
\end{array}\right. \\
& \left.\rho u_{i} u_{2}+\delta_{i, 2} p \quad \rho u_{i} u_{3}+\delta_{i, 3} p \quad(\rho e+p) u_{i}\right] \\
& D_{i}=\left[\begin{array}{llll}
\rho_{1} v_{D 1, i} & \rho_{2} v_{D 2, i} & \cdots & \rho_{n s} v_{D n s, i}
\end{array}\right. \\
& \left.\tau_{i, 1} \quad \tau_{i, 2} \quad \tau_{i, 3} \quad u_{j} \tau_{i, j}+q_{i}\right], \quad j=1,2,3 \\
& S=\left[\begin{array}{llllllll}
\dot{\omega}_{1} & \dot{\omega}_{2} & \cdots & \dot{\omega}_{n s} & 0 & 0 & 0 & 0
\end{array}\right]
\end{aligned}
$$

In the above equations, the total density of the mixture $\rho=\sum_{1}^{n s} \rho_{s p}$, and the partial density of the species $s p(s p=1,2, \cdots n s)$ are denoted by $\rho_{s p}$. $u_{i}(i=1,2,3)$ are velocity components in $\mathrm{x}, \mathrm{y}$ and $\mathrm{z}$ directions. $e$ and $p$ denote the total energy and the pressure of the mixture respectively. $v_{D s p, i}$ represents the diffusion velocity of the species $s p$ in $x_{i}$ direction. $\tau$ and $q$ define the viscous stress and heat conduction terms, and $\dot{\omega}_{s p}$ is the variation rate of the species $s p$. The transport properties of each pure component, such as viscosity and thermal conductivity, and thermodynamic properties, such as special heat capacity and enthalpies, are obtained from relations given in the power series form of temperature. The effective binary diffusion model rather than Fick's model is applied to approximate the diffusion velocities of all species. For the chemical kinetic model, 21 elementary reactions and 10 species, $\mathrm{I}_{2}, \mathrm{I}_{2}{ }^{*}, \mathrm{I}, \mathrm{I}^{*}, \mathrm{O}_{2}\left({ }^{1} \triangle\right), \mathrm{O}_{2}\left({ }^{1} \Sigma\right)$, $\mathrm{O}_{2}\left({ }^{3} \sum\right), \quad \mathrm{H}_{2} \mathrm{O}, \quad \mathrm{cl}_{2}$ and $\mathrm{N}_{2}$ are taken into consideration. The transport property model, thermodynamics definitions, diffusion model and chemical reactions were synthetically analyzed and presented (Paschkewitz et al., 2000; Eppard et al., 2000).

The total energy per specific volume is defined as:

$$
\rho e=\rho h-p+\rho u_{i} u_{i} / 2, \quad i=1,2,3
$$

The specific heat, enthalpy and entropy for each species can be calculated by the following polynomials:

$$
\begin{aligned}
& C p_{s p} / R_{s p}=\frac{a_{1 s p}}{T^{2}}+\frac{a_{2 s p}}{T}+a_{3 s p}+a_{4 s p} T \\
& +a_{5 s p} T^{2}+a_{6 s p} T^{3}+a_{7 s p} T^{4}+\frac{a_{8 s p}}{T^{3}} \\
& \frac{h_{s p}}{R_{s p} T}=-\frac{a_{1 s p}}{T^{2}}+a_{2 s p} \frac{\ln T}{T}+a_{3 s p}+\frac{a_{4 s p}}{2} T+\frac{a_{5 s p}}{3} T^{2} \\
& +\frac{a_{6 s p}}{4} T^{3}+\frac{a_{7 s p}}{5} T^{4}-\frac{a_{8 s p}}{2 T^{3}}+\frac{a_{9 s p}}{T} \\
& \frac{S_{s p}}{R_{s p}}=-\frac{a_{1 s p}}{2 T^{2}}-\frac{a_{2 s p}}{T}+a_{3 s p} \ln T+a_{4 s p} T+\frac{a_{5 s p}}{2} T^{2} \\
& +\frac{\mathrm{a}_{6 \mathrm{sp}}}{3} \mathrm{~T}^{3}+\frac{\mathrm{a}_{7 \mathrm{sp}}}{4} \mathrm{~T}^{4}+\frac{\mathrm{a}_{8 \mathrm{sp}}}{3 \mathrm{~T}^{3}}+\mathrm{a}_{10 \mathrm{sp}}
\end{aligned}
$$

In equations (2.1) to (2.3), parameters $a_{1}$ to $a_{10}$ are coefficients for calculating thermodynamic properties of individual species and can be found in the work of Paschkewitz et al.(2000). Therefore, the enthalpy of the mixture, $h$, is

$$
h=\sum_{s p=1}^{n_{s}} c_{s p} h_{s p}
$$

where $c_{s p}=\rho_{s p} / \rho$ is the mass fraction of species $s p$. According to Dalton's law, pressure $p$ is

$p=\sum_{s p=1}^{n_{s}} \rho_{s p} R_{s p} T$

where, $R$ is the gas constant and $T$ is the mixture temperature.

The thermal conductivity coefficient and the viscosity of each pure species can be approximated by the following polynomials:

$$
\begin{aligned}
& \kappa=a+b T+c T^{2}+d T^{3} \\
& \mu=A+B T+C T^{2}
\end{aligned}
$$

The coefficients, $a, b, c, d, A, B$, and $C$, for calculating transport properties in equations (3.1) and (3.2) can be found in a relevant literature (Paschkewitz et al., 2000). And the transport coefficient, $\eta=(\kappa, \mu)$ of the mixture is given by Wilke's equations: 
$\eta_{m i x}=\sum_{i=1}^{n s} \frac{\chi_{i} \eta_{i}}{\sum_{j=1}^{n s} \chi_{j} \phi_{i j}}$

$\phi_{i j}=\left(1+\left[\eta_{i} / \eta_{j}\right]^{0.5}\left[w_{j} / w_{i}\right]^{0.25}\right)^{2} /\left(8\left[1+w_{i} / w_{j}\right]\right)^{0.5}$

where, the molecular weight and the molar fraction of each species are denoted by $w$ and $\chi$.

An accurate description of the molecular diffusion of chemical species is important in the low pressure flowfields of chemical lasers. The RamshawDukowicz approximation for multi-component diffusive transport is used, including concentrationgradient-driven and pressure-gradient-driven contributions:

$\rho_{s p} v_{s p}=-\gamma_{t}\left[G_{s p}^{x}-\frac{\rho_{s p}}{\rho} \sum_{j=1}^{n s} G_{j}^{x}\right]-\gamma_{t}\left[G_{s p}^{p}-\frac{\rho_{s p}}{\rho} \sum_{j=1}^{n s} G_{j}^{p}\right]$

$G_{s p}^{x}=w_{s p} D_{s p} \nabla \chi_{s p}$

$G_{s p}^{p}=w_{s p} D_{s p}\left(\chi_{s p}-\rho_{s p} / \rho\right) \nabla P / P$

Here, $\gamma_{t}$ is the total molar concentration. The average diffusion coefficient of species $D_{s p}$ is calculated by a binary effective diffusion model.

The production rate of species $s p$ is determined by

$\dot{\omega}_{s p}=w_{s p} \sum_{r=1}^{N R}\left[\left(v_{s p, r}^{\prime}-v_{s p, r}^{\prime \prime}\right)\right.$

$\left.\left(k_{f, r} \prod_{j=1}^{n_{s}}\left(\chi_{j}\right)^{\gamma_{j, r}^{\prime}}-k_{b, r} \prod_{j=1}^{n_{s}}\left(\chi_{j}\right)^{\gamma^{\prime \prime}, r}\right)\right]$

The forward reaction rate constant, $k_{f, r}$, of each reaction is calculated by the Arrhenius equation and the corresponding backward reaction rate constant, $k_{b, r}$, can be derived from the equilibrium coefficient, $k_{e, r}$, and the forwards rate constant:

$k_{f, r}=C_{r} T^{n r} \exp \left(-E a_{r} / R T\right)$

$\kappa_{b, r}=\frac{\kappa_{f, r}}{\kappa_{e, r}}$

$$
\begin{aligned}
\kappa_{e, r}= & \exp \left(\sum_{s p=1}^{n s}\left[\left(v_{s p, r}^{\prime \prime}-v_{s p, r}^{\prime}\right)\left(\frac{s_{s p}}{R_{s p}}-\frac{h_{s p}}{R_{s p} T}\right)\right]\right) \\
& \left(\frac{P_{a t m}}{R T}\right)^{\sum_{s p=1}^{n s}\left(v_{s p, r}^{\prime \prime}-v_{s p, r}^{\prime}\right)}
\end{aligned}
$$

In these equations, $v_{s p, r}^{\prime}$ and $v_{s p, r}^{\prime \prime}$ are the stoichiometric coefficient of species $s p$ in the $r$ th elementary reaction as the reactant and the product, respectively. The pre-exponential factor, $C_{r}$, the temperature index, $n r$, and the activation energy, $E a_{r}$ are given by the detailed finite-rate chemical reaction model. The main reaction kinetic processes can be illustrated through Fig. 1. Reaction equations (1) to (4) model the self-catalyzed chain reaction of the dissociation of $I_{2}$, where equation (1) is the primary onset reaction and equation (3) represents the resonant energy transfer or pumping reaction. Equations (5), (6) and (7) denote different sorts of quench processes by third-bodies. Equation (8) is the stimulated laser radiation. For simplicity, other reaction equations are not included in Fig. 1 but can be found in the work of Paschkewitz et al. (2000).

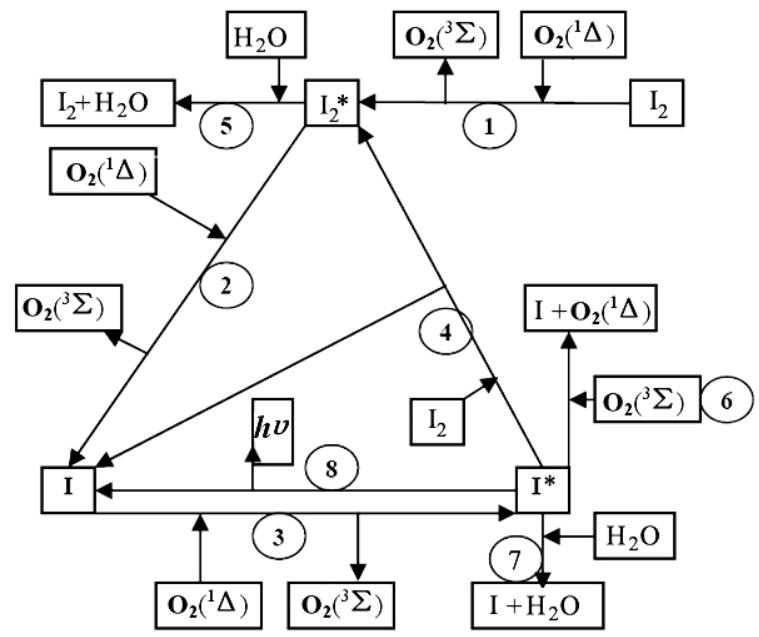

Fig. 1 Sketch of the kinetic processes in the COIL mixing nozzle.

\subsection{Numerical algorithms}

The semi-discretized difference equation for equation (1) is

$\left(\frac{\partial \mathrm{U}}{\partial \mathrm{t}}\right)=-\mathrm{CONV}_{\mathrm{i}, \mathrm{j}, \mathrm{k}}^{\mathrm{n}}+\mathrm{VISC}_{\mathrm{i}, \mathrm{j}, \mathrm{k}}^{\mathrm{n}}+\mathrm{S}_{\mathrm{i}, \mathrm{j}, \mathrm{k}}^{\mathrm{n}}$ 
The dissipative term, VISC, is discretized by a second-order centered difference scheme, while the convective term, CONV, is discretized by using a second-order scheme, DCD (Dispersion-controlled dissipative) scheme (Jiang, Takayama and Chen, 1995; Jiang, 2004; Hu et al., 2004) proposed by Jiang et al. for shock-wave-capturing. The computational domains used in present simulations were all discretized on body-fitted, structured grids, with local mesh refinement near the jet regions and the solid boundaries.

DCD scheme uses the Steger-Warming (Steger and Warming, 1981) flux splitting algorithm. In the general coordinate system $(\xi, \eta, \zeta)$, the split convective flux vectors are simple and symmetrical. Here is the split convective flux vector of flux $\widetilde{F}$ in $\xi$ direction:

$$
\widetilde{F}^{ \pm}=\frac{\rho}{2 J \hat{\gamma}}\left[\begin{array}{c}
C_{1}\left[2(\hat{\gamma}-1) \widetilde{\lambda}_{1}^{ \pm}+\widetilde{\lambda}_{n s+3}^{ \pm}+\widetilde{\lambda}_{n s+4}^{ \pm}\right] \\
\vdots \\
C_{n s}\left[2(\hat{\gamma}-1) \widetilde{\lambda}_{1}^{ \pm}+\widetilde{\lambda}_{n s+3}^{ \pm}+\widetilde{\lambda}_{n s+4}^{ \pm}\right] \\
v\left[2(\hat{\gamma}-1) \widetilde{\lambda}_{1}^{ \pm}\right]+\left(u-c k_{x}\right) \widetilde{\lambda}_{n s+3}^{ \pm}+\left(u+c k_{x}\right) \widetilde{\lambda}_{n s+4}^{ \pm} \\
v\left[2(\hat{\gamma}-1) \widetilde{\lambda}_{1}^{ \pm}\right]+\left(v-c k_{y}\right) \widetilde{\lambda}_{n s+3}^{ \pm}+\left(v+c k_{y}\right) \widetilde{\lambda}_{n s+4}^{ \pm} \\
w\left[2(\hat{\gamma}-1) \widetilde{\lambda}_{1}^{ \pm}\right]+\left(w-c k_{z}\right) \tilde{\lambda}_{n s+3}^{ \pm}+\left(w+c k_{z}\right) \widetilde{\lambda}_{n s+4}^{ \pm} \\
2\left[(\hat{\gamma}-1) H-c^{2}\left[\widetilde{\lambda}_{1}^{ \pm}+(H-c \theta) \widetilde{\lambda}_{n s+3}^{ \pm}+(H+c \theta) \widetilde{\lambda}_{n s+4}^{ \pm}\right.\right.
\end{array}\right]
$$

where $\hat{\lambda}_{i}^{ \pm}=\frac{1}{2}\left(\hat{\lambda}_{i} \pm \sqrt{\hat{\lambda}_{i}^{2}+\varepsilon^{2}}\right) \quad i=1,2, \cdots n s+4$, and $\varepsilon$ is very small number to keep all the eigenvalues nonzero. $\theta=u k_{x}+v k_{y}+w k_{z}$, $k_{x}=\xi_{x} / \Delta \quad, \quad k_{y}=\xi_{y} / \Delta, \quad k z=\xi_{z} / \Delta$, $\Delta=\sqrt{\xi_{\mathbf{x}}^{2}+\xi_{\mathbf{y}}^{2}+\xi_{\mathbf{z}}^{2}}$. Here, $\xi_{x}, \xi_{y}, \xi_{z}$ are transformation matrices between the physical and computational space, and $J$ is the Jacobian of the transformation.

An explicit and second-order Runge-Kutta integration method is used to advance the computational solution in time toward steady-state. The convergence criterion is defined as $\operatorname{Re} s=\frac{\dot{m}_{\text {in }}-\dot{m}_{\text {out }}}{\dot{m}_{\text {in }}} \leq 10^{-4}$, where $\dot{m}_{\text {in }}$ and $\dot{m}_{\text {out }}$ are the mass flow rates of some important species at the inflow and outflow surface of the nozzle respectively, for example, helium for the validation case and oxygen for other cases. One run took about a month on a self-parallelized 64-bit workstation with 4-processors.

\section{NUMERICAL RESULTS AND DISCUSSIONS}

\subsection{Validation}

To verify the numerical algorithms and validate the numerical solutions, a cold flow with a transonic injection in a RADICL (research assessment device improvement chemical laser) slit nozzle has been studied. The geometry used in the simulation was based on the experimental device by Miller et al. (2000). The complete RADICL nozzle has 115 large injectors and 230 small injectors on either side of the nozzle blade, and the nominal nozzle width is $25.4 \mathrm{~cm}$. The computational domain used the unit cell approximation based on the symmetry of the RADICL nozzle geometry. The symmetry planes include the nozzle centerline. The centerline passes through the large injector and the symmetry line bisects the distance between two adjacent small injectors. In other words, the computational domain is a $1 / 460$ cut of the entire nozzle flowfield. Fig. 2(a) shows the computational domain and the enlargement of the injectors of two symmetric unit cells. A coarse grid $(601 \times 71 \times 31)$ and a fine grid $(601 \times 91 \times 41)$ were used to discretize the nozzle. The flow conditions were set according to the experimental setup in the study of Miller et al. (2000). Firstly, a secondary flow was perpendicularly injected into the primary flow near the nozzle throat. Secondly, helium was used for both the primary and secondary flows. Then we used a total temperature and pressure of $T_{p}=293 \mathrm{~K}$ and $\mathrm{p}_{\mathrm{p}}=7955 \mathrm{~Pa}$ for the primary flow (p) and $\mathrm{T}_{\mathrm{s}}=403 \mathrm{~K}$ and $\mathrm{p}_{\mathrm{s}}=32925 \mathrm{~Pa}$ for the secondary flow (s).

Fig. 2(b) shows computed wall pressure together with the measured experimental data. There is a good agreement between the numerical results for both the coarse and fine grids and the experiment. The discrepancy in the pressure measurements is highest near the downstream nozzle exit, which is considered to be a consequence of injecting a purge flow in the $\mathrm{z}$ direction downstream of the nozzle throat in the experiment (Miller et al., 2000). The application of the fine grid causes a minor change in the wall pressure. From a direct comparison of these results, it can be concluded that the numerical solutions are well validated for the jet flow in the COIL. 

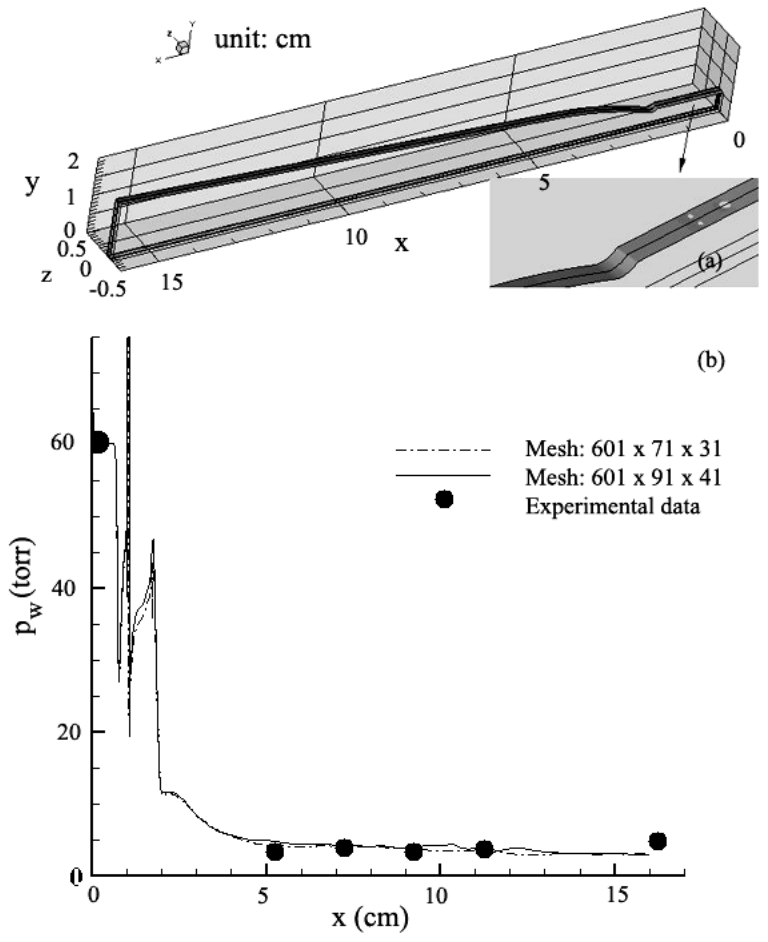

Fig. 2 RADICL slit nozzle flow simulation for validation and grid-independence analysis: (a) computational domain and (b) comparison between the numerical results and experimental data in wall pressure.

\subsection{Computational setup for the transverse interleaved jet}

Downstream from the throat, two rows of jet orifices are located on either side of the nozzle blade. The jet orifices, which have a diameter of $1 \mathrm{~mm}$, are interleaved and set $2 \mathrm{~mm}$ apart from each other in the $\mathrm{z}$ direction. The height of the nozzle throat is $6 \mathrm{~mm}$. The angle between the secondary jets and the nozzle axis is $45^{\circ}$. Fig. 3(a) shows the computational domain - the unit cell approximation enveloped by the thick lines, which include a half jet orifice on either side of the nozzle blade. Fig. 3(b) and (c) demonstrate the jet arrangement. Total grid point of $501 \times 121 \times 31$ is used to discretize the domain, while mesh refinement is handled near the jets and the nozzle walls. The secondary flow is diluted by the buffer gas $\mathrm{N}_{2}$. Two cases were considered, each with a different molar ratio of $\mathrm{I}_{2} / \mathrm{N}_{2}$ : namely case (1) $1 / 60$ and case (2) $2 / 59$. The total flow rate of the secondary jets for the computational unit, $\dot{n}_{s}$, is $1.86 \times 10^{-5} \mathrm{~mole} / \mathrm{s}$, and the stagnation temperature, $\mathrm{T}_{\mathrm{s} 0}$, is $383 \mathrm{~K}$. For both cases considered, the molar ratio of the primary flow, $\mathrm{O}_{2}\left({ }^{3} \sum\right) / \mathrm{O}_{2}\left({ }^{1} \Delta\right) / \mathrm{H}_{2} \mathrm{O} / \mathrm{Cl}_{2}$, is given as $1 / 1.5 / 0.278 / 0.278$. The primary flow rate for the computational unit, $\dot{n}_{p}$, is $0.99 \times 10^{-5} \mathrm{~mole} / \mathrm{s}$, while $\mathrm{T}_{\mathrm{p} 0}=271.3 \mathrm{~K}$.
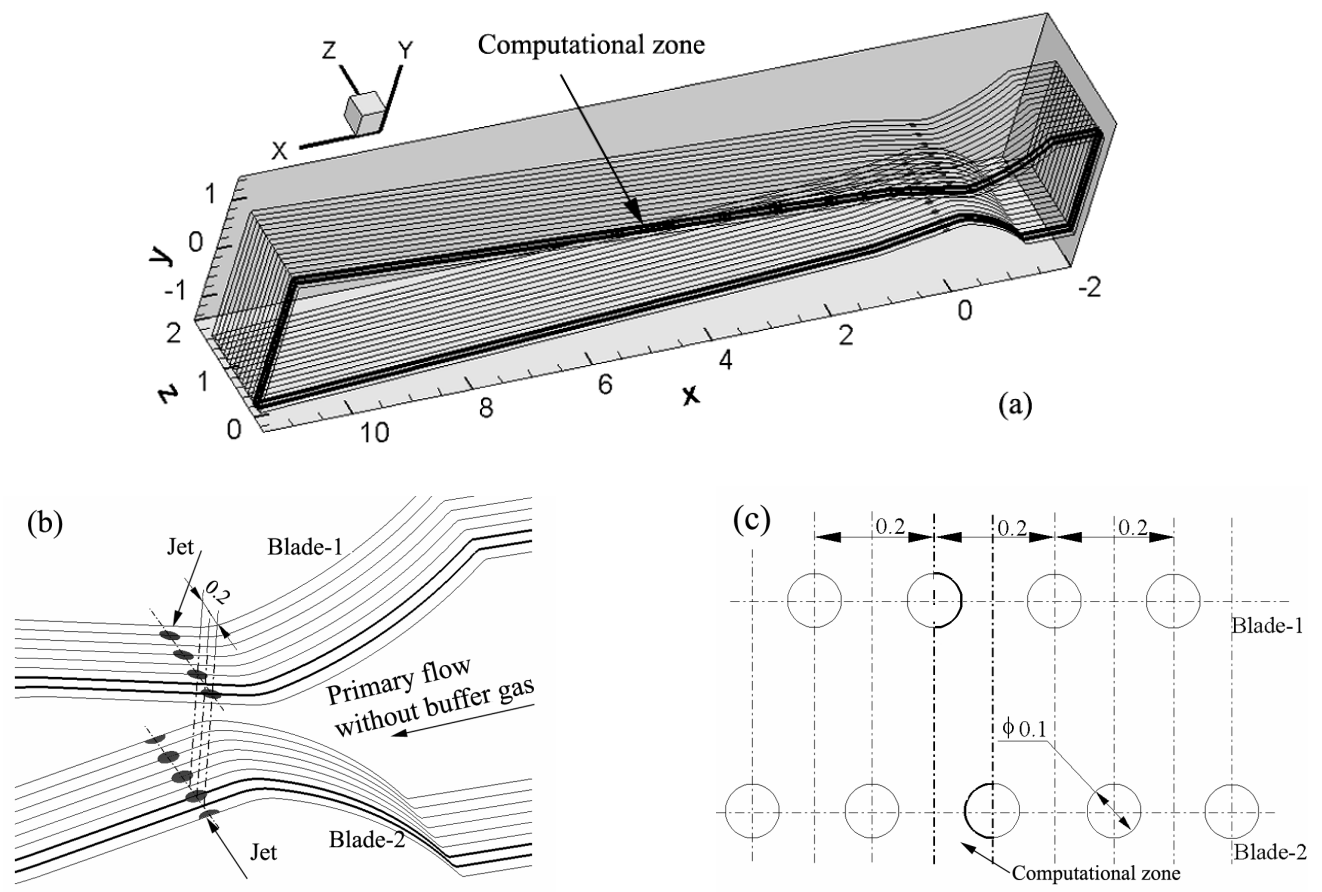

Fig. 3 (a) Computational domain for the interleaved jet configuration in the supersonic section of the COIL nozzle; (b) Enlargement of the jet region; (c) Arrangement of the jet orifices (length unit: $\mathrm{cm}$ ). 


\subsection{Features of the flowfield}

For the jet mixing in a COIL systems operating without primary buffer gas, the under-expanded secondary flows are always injected from the orifices located in the transonic region downstream from the nozzle throat. Generally, the injection of gas into the supersonic flow induces bow shock waves in front of the jet trajectories. If the structures block the stream to a certain extent, the geometric throat doesn't work and a gasdynamic throat takes over the responsibility of the subsonicsupersonic transition. Fig. 4 show the flowfield in the $\mathrm{z}=0$ symmetric plane of the upper jet orifice. In Fig. 4(a), an irregular gasdynamic throat can be seen, as well as a barrel shock and a Mach disc associated with the under-expanded jet condition. The interleaved jets result in an asymmetric flowfield with the sharp gradients of velocity, temperature and species concentration. Fig. 4(b)
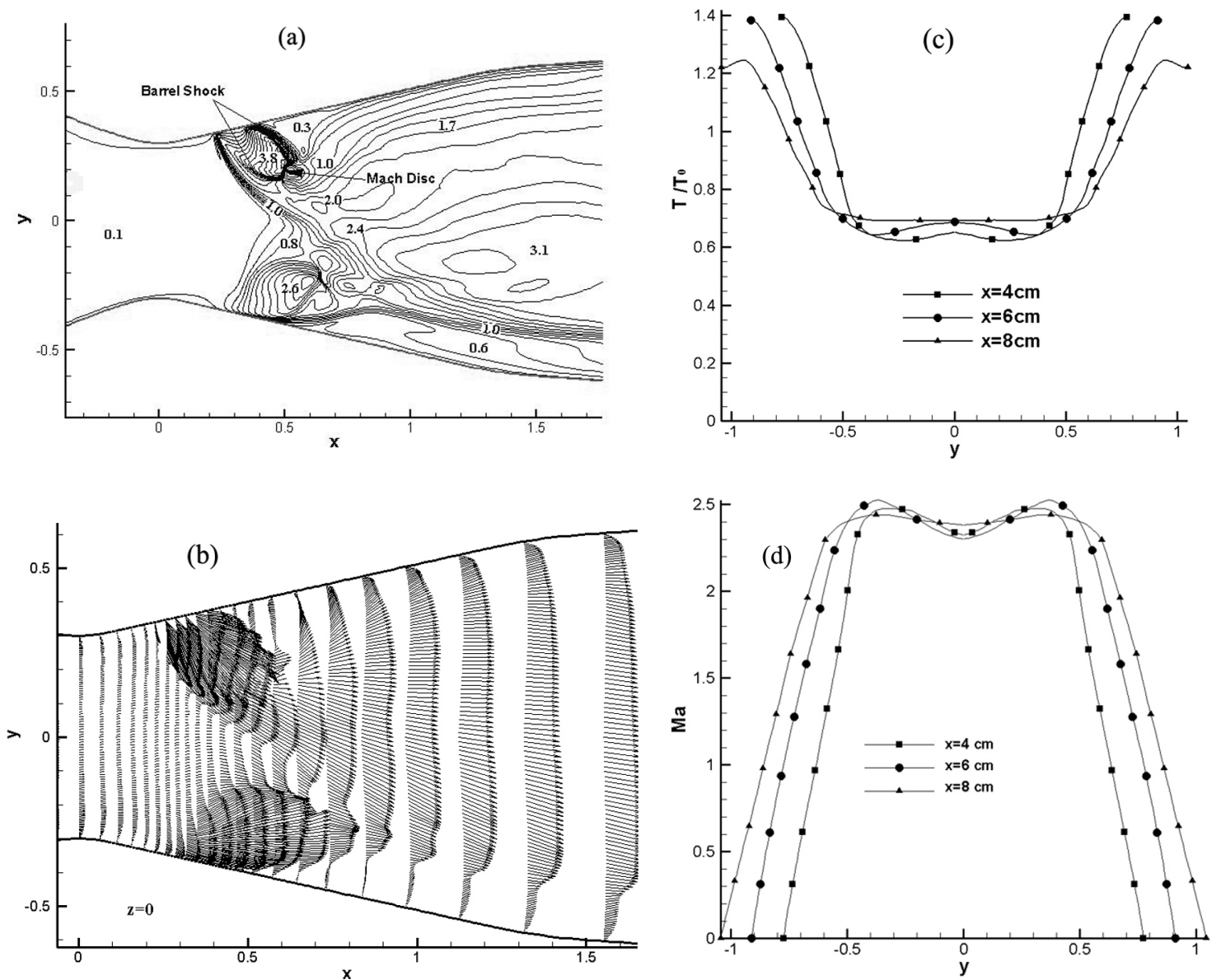

Fig. 4 Flow structures in the near field of the jets: (a) Mach number, (b) two-dimensional flow vector in the symmetric plane of the upper jet orifice (the flow direction is from left to right), (c) temperature distribution and (d) Mach number in the z-symmetric plane (length unit: $\mathrm{cm}$ ). 


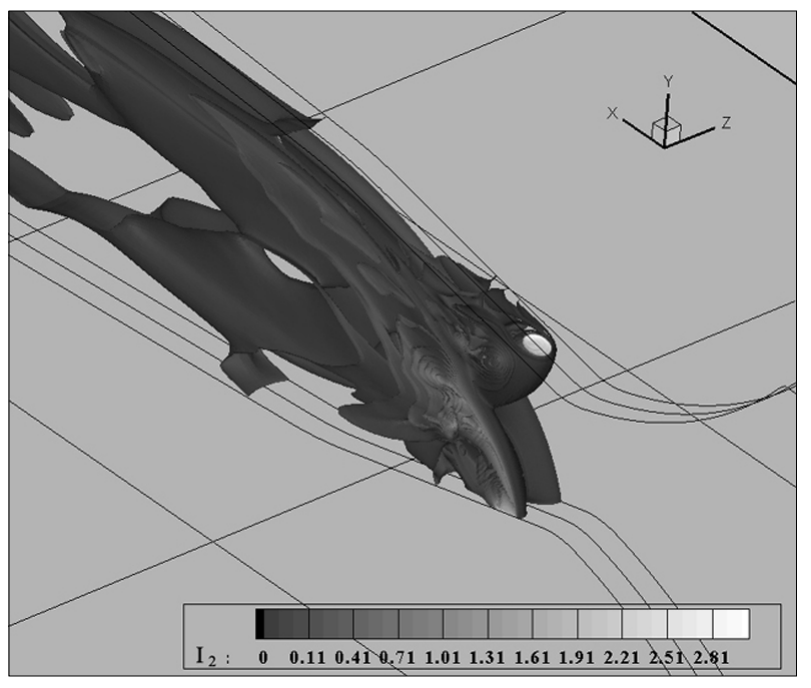

Fig. 5 Three-dimensional distribution of $\mathrm{I}_{2}$ density (normalized by the primary flow density).

The distribution contour of molecular iodine is given in Fig. 5. In this figure, one can find the three-dimensional structures of the under-expanded jet and the interaction among the interleaved jet trajectories. The blockage induced by the jet structures should be considered carefully. The subsonic jet mixing configurations which were used in the traditional COIL design may worth further studying for COIL operating without primary buffer gas even though they were not recommended in preceding research (Fang, Sang and Chen, 2002 \& 2003).

The distribution of several species is shown in Fig. 6 for case (1) and case (2). The molecular iodine maintains mostly in the near field of jets as indicated in Fig. 6(a) and (b), while in the richiodine case the iodine molecules extends more deeply into the downstream field of the nozzle flow than that in case (1). As shown in Fig. 6(c) and (f), the interleaved jets cause the heterogeneous distributions of laser media, the excited and ground state iodine. Comparing the distributions of $I^{*}$ in
Fig. 6(c) and (d), the rich-iodine condition results in a higher concentration of $I^{*}$ in the core flow than that in case (1). Within both the jet trajectories, there are two strip-like regions which are abundant in ground state iodine atom as seen in Fig. 6(e) and (f). Such regions indicate that the chain reactions of dissociation illustrated by Fig. 1 go on successfully benefiting from the sufficient molecular iodine within the jet trajectories and efficient mixing between the singlet oxygen flow and the jet flow. Just in the same region, however, I* is infrequent as shown in Fig. 6(d), which is ultimately different from the more homogeneous distribution of $I^{*}$ in Fig. 6(c). The nonlinear effects associated with the concentration of the molecular iodine fed by jet flows can hardly be predicted by using any analytical methods. The three-dimensional CFD is helpful to gain understanding of the physical and chemical processes which take place in the COIL mixing nozzles.

\subsection{Spatial distribution of the small signal gain}

The distribution of the small signal gain for the two cases is plotted in Figs. 7. In Fig. 7(a), the small signal gain degenerates to zero in the boundary layers, which letdowns the laser energy density. The spanwise distribution of the small signal gain in case (1) appears more reasonable than that of case (2) as shown in Fig. 7(b). Negative gain strips along the jet trajectories can be seen in Fig. 7(b). The distributional disparity between $\mathrm{I}^{*}$ and I shown in Fig. 6(d) and (f) accounts for this negative gain since the small signal gain is proportional to the molar concentration difference, $\left(\left[I^{*}\right]-0.5[I]\right)$. The details of the spatial distribution and the evolution of the small signal gain will be explained in a follow-up research article in the future. 

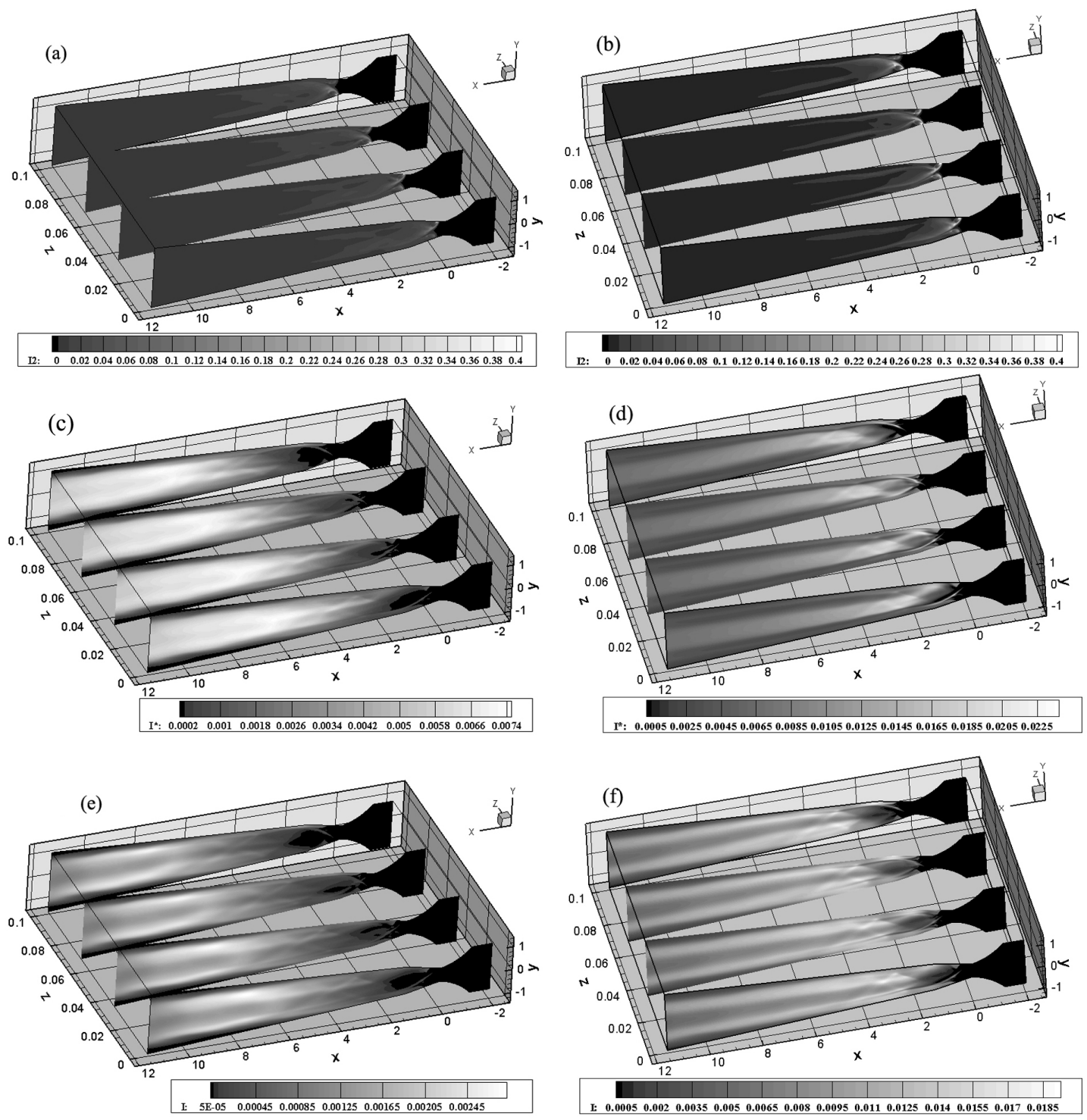

Fig. 6 Distribution of species in the longitudinal planes: molecular iodine for (a) case 1 and (b) case 2; pumped iodine atom for (c) case 1 and (d) case 2; ground-state iodine atom for (e) case 1 and (f) case 2 (length unit: cm, density normalized by the primary flow density).
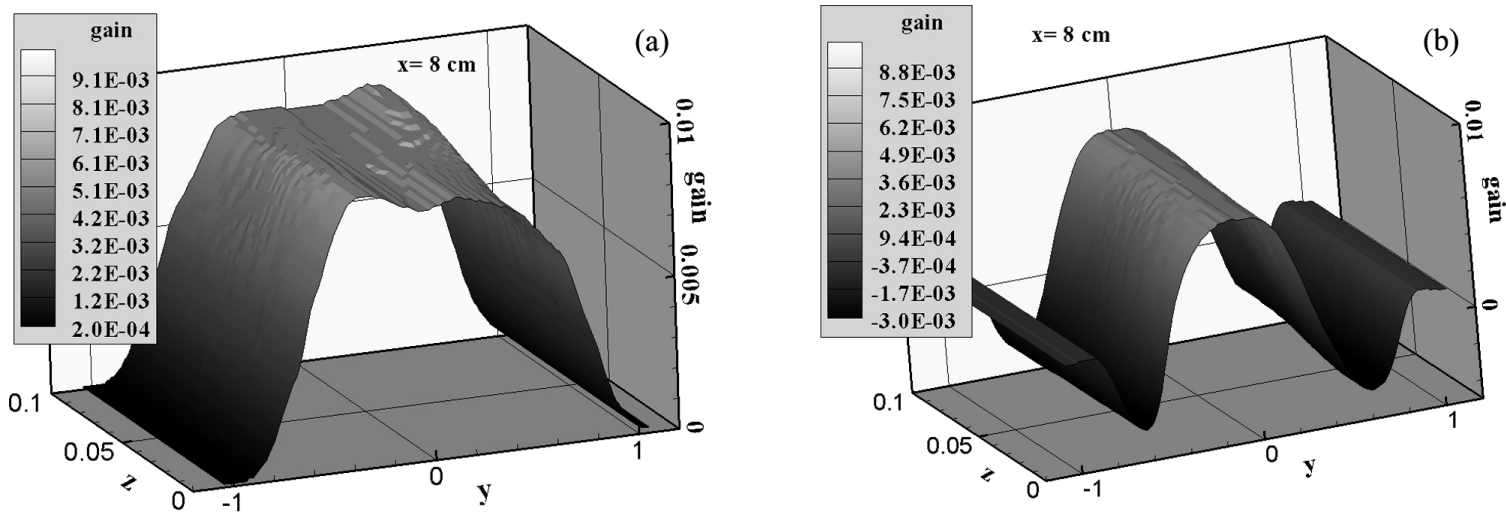

Fig. 7 Distribution of the small signal gain in $y-z$ planes $\left(x=8 \mathrm{~cm}\right.$ ): (a) case $1-\mathrm{I}_{2} / \mathrm{N}_{2}=1 / 60$ and (b) case $2-\mathrm{I}_{2} / \mathrm{N}_{2}=2 / 59$ (gain unit: $\mathrm{cm}^{-1}$, length unit: $\mathrm{cm}$ ). 


\section{CONCLUSIONS}

Through the numerical studies of the mixing and reactive flow in the mixing nozzle of COIL operating without primary buffer gas, some important observations are summarized as follows: A reasonable distribution and spatial evolution of the small signal gain can be obtained through the interleaved injection scheme if a proper molecular iodine flow rate is used in the mixing nozzle. The laser medium distribution suffers from the iodine flow rate because of the nonlinear interaction between the fluid dynamics of jets and the chain reaction kinetics of the self-catalyzed $\mathrm{I}_{2}$ dissociation.

\section{ACKNOWLEDGEMENTS}

The authors would like to thank Prof. B.G. Wu and Prof. G.W. Yang at the Institute of Mechanics. This work was partially supported by Korea Research Foundation Grant No. KRF-2005-005-J09901.

\section{REFERENCES}

1. Bruins E, Furman D, Rybalkin V, Barmashenko $\mathrm{BD}$ and Rosenwaks S (2002). One-dimensional modeling of the gain and temperature in a supersonic chemical oxygen-iodine laser with transonic injection of iodine. IEEE Journal of Quantum Electronics 38(4):345-352.

2. Eppard WM, McGrory WD, Godfrey AG, Cliff EM and Borggaard JT (2000). Recent advances in numerical techniques for the design and analysis of COIL system. AIAA Paper 2000-2576. 31st AIAA Plasmadynamics and Lasers Conference, 19-22 June 2000, Denver, CO, USA.

3. Fang BJ, Sang FT and Chen F (2002). Experimental study on kW COIL with nitrogen buffer gases. High Power Laser and Particle Beam 14(3):447-450.

4. Fang BJ, Sang FT and Chen F (2003). Design and experimental results of $2 \mathrm{~kW}$ COIL with nitrogen buffer gases. High Power Laser and Particle Beam 15(12):1148-1150.

5. Furman D, Barmashenko BD and Rosenwaks $\mathrm{S}$ (1998). Parameter study of an efficient supersonic chemical oxygen-iodine laser/jet generate system operating without buffer gas. IEEE Journal of Quantum Electronics 34:1068-1074.
6. Furman D, Bruins E, Rybalkin V, Barmashenko BD and Rosenwaks S (2001). Parameter study of small-signal gain in s slit nozzle, supersonic chemical oxygen-iodine laser operating without primary buffer gas. IEEE Journal of Quantum Electronics 37(2):174-182.

7. $\mathrm{Hu} \mathrm{ZM}$, Gao YL, Zhang DL, Yang GW and Jiang ZL (2004). Numerical simulation of gaseous detonation reflection on wedges with a detailed chemical reaction model. Acta Mechanica Sinica 36(4):385-392 (Chinese series).

8. Jiang ZL, Takayama K and Chen YS (1995). Dispersion conditions for non-oscillatory shock capturing schemes and its applications. Computational Fluid Dynamics Journal 4:137-150.

9. Jiang ZL (2004). On dispersion-controlled principles for non-oscillatory shock-capturing schemes. Acta Mechanica Sinica 20(1):1-15.

10. Miller JH, Shang JS, Tomaro RF and Strang WZ (2000). Computation of COIL nozzle flowfields with transonic injection. AIAA Paper 2000-2575. 31st AIAA Plasmadynamics and Lasers Conference, 19-22 June 2000, Denver, CO, USA.

11. Paschkewitz J, Shang J, Miller J and Madden T (2000). An assessment of COIL physical property and chemical kinetic modelling methodologies. AIAA Paper 2000-2574. 31st AIAA Plasmadynamics and Lasers Conference, 19-22 June 2000, Denver, CO, USA.

12. Rybalkin V, Katz A, Furman D, Barmashenko BD and Rosenwaks S (2002). Spatial distribution of the gain and temperature across the flow in a slit-nozzle supersonic chemical oxygen-iodine laser with transonic and supersonic schemes of iodine injection. IEEE Journal of Quantum Electronics 38(10):1398-1405.

13. Steger JL and Warming RF (1981). Flux vector splitting of the inviscid gas dynamic equations with applications to finite difference method. Journal of Computational Physics 40:263-293. 